\title{
Decoherence-Free Quantum Information Processing with Four-Photon Entangled States
}

\author{
Mohamed Bourennane ${ }^{1,2}$, Manfred Eibl ${ }^{1,2}$, Sascha Gaertner ${ }^{1,2}$, Christian Kurtsiefer ${ }^{2}$, \\ Adán Cabello ${ }^{3}$, and Harald Weinfurter ${ }^{1,2}$ \\ ${ }^{1}$ Max-Planck-Institut für Quantenoptik, D-85748 Garching, Germany \\ ${ }^{2}$ Sektion Physik, Ludwig-Maximilians-Universität, D-80797 München, Germany \\ ${ }^{3}$ Departamento de Física Aplicada II, Universidad de Sevilla, E-41012 Sevilla, Spain
}

\begin{abstract}
Decoherence-free states protect quantum information from collective noise, the predominant cause of decoherence in current implementations of quantum communication and computation. Here we demonstrate that spontaneous parametric down-conversion can be used to generate four-photon states which enable the encoding of one qubit in a decoherence-free subspace. The immunity against noise is verified by quantum state tomography of the encoded qubit. We show that particular states of the encoded qubit can be distinguished by local measurements on the four photons only.
\end{abstract}

PACS numbers: 03.67.Pp, 03.65.Yz, 03.67.Hk, 42.65.Lm

Quantum information processing enables secure classical communication, powerful quantum communication schemes, and speedup in computation [1]. These methods rely on the preparation, manipulation, and detection of the superposition of quantum states. Superpositions, however, are very fragile and easily destroyed by the decoherence processes due to unwanted coupling with the environment 2]. Such uncontrollable influences cause noise in the communication or errors in the outcome of a computation, and thus reduce the advantages of quantum information methods.

Several strategies have been devised to cope with decoherence, each of them appropriate for a specific type of coupling with the environment. For instance, if the interaction with the environment is weak enough such that qubits are affected only with a very low probability, a good strategy would be to add redundancy when encoding the quantum information in order to detect and correct the errors by active quantum error correction methods [3].

If the qubit-environment interaction, no matter how strong, exhibits some symmetry, then there exist quantum states which are invariant under this interaction. These states are called decoherence-free (DF) states, and allow protection of quantum information [4, 5, 6, 7]. A particularly relevant symmetry arises when the environment couples with the qubits without distinguishing between them, resulting in the so-called collective noise. This situation occurs, for instance, when the spatial (temporal) separation between the carriers of the qubits is small relative to the correlation length (time) of the environment. Typical examples arise in ion-trap or nuclear magnetic resonance (NMR) experiments, which are susceptible to fluctuations of magnetic or electrostatic fields, but also in quantum communication, e.g., when the qubits carried by polarized photons are successively sent via the same optical fiber and therefore experience the same birefringence.
Experimental efforts investigating features of DF systems so far have been limited to two-qubit systems only. For two qubits, however, the singlet state is the only DF state and thus, it is not sufficient to fully protect an arbitrary logical qubit against collective noise. The experiments so far have demonstrated the features of the single DF state or the immunity against restrictive types of noise [8]. For three qubits there is no DF state immune to collective noise. However, quantum information can be preserved in an abstract subsystem known as a noiseless subsystem, which was demonstrated in NMR experiments $[9]$.

In this Letter we report on the production of various decoherence-free four-photon polarization-entangled states using spontaneous parametric down conversion (SPDC). The immunity of these states against collective noise is experimentally verified by showing their invariance when passing the four photons through a noisy environment simulated by birefringent media. Moreover, we show that one can both distinguish two orthogonal four-photon DF states and perform state tomography by local polarization measurements only. We are thus able to demonstrate that quantum information can be reliably extracted from qubits communicated through a noisy environment, and also between parties who do not even share a common reference frame.

For the construction of DF states formed by $N$ qubits, we note that for collective noise, all $N$ qubits undergo the same (unknown) unitary transformation $U$. States are decoherence-free if they are invariant under such a $N$-lateral unitary transformation, i.e., $U^{\otimes N}|\psi\rangle=|\psi\rangle$, where $U^{\otimes N}=U \otimes \ldots \otimes U$ denotes the tensor product of $N$ unitary transformations $U[$ [] $]$. The amount of quantum information that a given DF subspace is able to protect is determined by the number $N$ of qubits used [5]. For $N=2$ qubits there is only one DF state, the singlet state, $\left|\psi^{-}\right\rangle_{a b}=(1 / \sqrt{2})(|01\rangle-|10\rangle)_{a b}$, where $|01\rangle_{a b}=|0\rangle_{a} \otimes|1\rangle_{b}$. The smallest useful DF subspace is spanned by two four- 
qubit DF states. We can choose one of them as the tensor product of two singlet states,

$$
\left|\Phi_{0}\right\rangle_{a b c d}=\left|\psi^{-}\right\rangle_{a b} \otimes\left|\psi^{-}\right\rangle_{c d}
$$

The DF state orthogonal to $\left|\Phi_{0}\right\rangle_{a b c d}$ is given by

$$
\begin{aligned}
\left|\Phi_{1}\right\rangle_{a b c d}= & \frac{1}{2 \sqrt{3}}(2|0011\rangle-|0101\rangle-|0110\rangle-|1001\rangle \\
& -|1010\rangle+2|1100\rangle)_{a b c d}
\end{aligned}
$$

and was first introduced by Kempe et al. 7].

The DF subspace spanned by the states $\left|\Phi_{0}\right\rangle$ and $\left|\Phi_{1}\right\rangle$ allows now to encode a qubit $|\Phi\rangle=c_{0}|0\rangle+c_{1}|1\rangle$ (where $c_{0}$ and $c_{1}$ are complex numbers) as the superposition state $\left|\Phi_{L}\right\rangle=c_{0}\left|\Phi_{0}\right\rangle+c_{1}\left|\Phi_{1}\right\rangle$, which is immune against collective noise for any $c_{0}$ and $c_{1}$. It is also important to be able to read the logical qubit $\left|\Phi_{L}\right\rangle$. This is usually carried out by projecting $\left|\Phi_{L}\right\rangle$ onto the basis states of the DF subspace requiring non trivial quantum gates. However, $\left|\Phi_{0}\right\rangle$ and $\left|\Phi_{1}\right\rangle$ can be distinguished using only local measurements: It suffices to project the first two qubits onto the computational basis $(|0\rangle$ and $|1\rangle)$ and the other two onto the Hadamard rotated basis $[|\overline{0}\rangle=(|0\rangle+|1\rangle) / \sqrt{2}$ and $|\overline{1}\rangle=(|0\rangle-|1\rangle) / \sqrt{2}]$. Expressed in these bases, the DF states read as

$$
\begin{aligned}
\left|\Phi_{0}\right\rangle= & (|01 \overline{1} \overline{0}\rangle-|01 \overline{0} \overline{1}\rangle+|10 \overline{0} \overline{1}\rangle-|10 \overline{1} \overline{0}\rangle) / 2 \\
\left|\Phi_{1}\right\rangle= & (|00 \overline{0} \overline{0}\rangle-|00 \overline{0} \overline{1}\rangle-|00 \overline{1} \overline{0}\rangle+|00 \overline{1} \overline{1}\rangle-|01 \overline{0} \overline{0}\rangle \\
& +|01 \overline{1} \overline{1}\rangle-|10 \overline{0} \overline{0}\rangle+|10 \overline{1} \overline{1}+| 11 \overline{0} \overline{0}\rangle+|11 \overline{0} \overline{1}\rangle\rangle \\
& +|11 \overline{1} \overline{0}\rangle+|11 \overline{1} \overline{1}\rangle) / 2 \sqrt{3}
\end{aligned}
$$

These states have no common terms and are therefore easily distinguishable using the outcomes of local measurements on the four qubits.

The invariance of the encoded quantum information is demonstrated best by comparing the density matrix $\rho_{L}=\left|\Phi_{L}\right\rangle\left\langle\Phi_{L}\right|$ of the logical qubit before $\left(\rho_{\text {in }}\right)$ and after $\left(\rho_{\text {out }}\right)$ the interaction with the environment. In order to evaluate the density matrix $\rho$ of an encoded qubit, one needs to measure 3 four-qubit observables $\Sigma_{z}, \Sigma_{x}$, and $\Sigma_{y}$. A well-suited choice is $\Sigma_{z}=\sigma_{z} \otimes \sigma_{z} \otimes \sigma_{x} \otimes \sigma_{x}$, $\Sigma_{x}=\sigma_{z} \otimes \sigma_{x} \otimes \sigma_{z} \otimes \sigma_{x}$, and $\Sigma_{y}=\sigma_{y} \otimes \sigma_{x} \otimes \sigma_{z} \otimes \mathbb{1}$, because they can be determined again by local measurements on the four photons. Here, $\left\{\sigma_{x}, \sigma_{y}, \sigma_{z}\right\}$ denote the Pauli matrices, and $\mathbb{1}$ is the identity. The results of these measurements allow us to perform the tomographic reconstruction of the density matrix $\rho$, since its elements can be expressed as $\rho_{11}=\left(3\left\langle\Sigma_{z}\right\rangle+1\right) / 4, \operatorname{Re}\left(\rho_{12}\right)=$ $\sqrt{3}\left(2\left\langle\Sigma_{x}\right\rangle+\left\langle\Sigma_{z}\right\rangle-1\right) / 4$, and $\operatorname{Im}\left(\rho_{12}\right)=\sqrt{3}\left\langle\Sigma_{y}\right\rangle / 2$, where $\left\langle\Sigma_{i}\right\rangle=\operatorname{Tr}\left(\rho \Sigma_{i}\right)$ describes the expectation value of $\Sigma_{i}$.

In our experiment the physical qubits are polarized photons, where the computational basis corresponds to horizontal and vertical linear polarization, "0" $\equiv H$ and "1" $\equiv V$. The four-photon polarization-entangled state $\left|\Phi_{0}\right\rangle$ can be obtained from two synchronized (first order)
SPDC sources for photon pairs in the singlet state $\left|\psi^{-}\right\rangle$. More practically, for the measurements shown in Figs. 2 and $3,\left|\Phi_{0}\right\rangle$ was generated by using the product state of two polarization-entangled photon pairs created from two consecutive pump pulses and swapping the modes $b \leftrightarrow c$. The four-photon polarization-entangled state $\left|\Phi_{1}\right\rangle$ was observed using the second order SPDC process [10, 11].

We used the UV pulses of a frequency-doubled modelocked Ti:sapphire laser (pulse length $130 \mathrm{fs}$ ) to pump a $2 \mathrm{~mm}$ thick BBO (barium betaborate) crystal at a wavelength of $390 \mathrm{~nm}$ and a repetition rate of $82 \mathrm{MHz}$ with an average power of $750 \mathrm{~mW}$ (see Fig. 1). The pump beam was focused to a waist of $100 \mu \mathrm{m}$ inside the crystal. The degenerate down-conversion emission into the two characteristic type-II crossing directions [12] was coupled into single mode optical fibers and passed through narrowband interference filters $(\Delta \lambda=3 \mathrm{~nm})$ to exactly define the spatial and spectral emission modes. To observe $\left|\Phi_{0}\right\rangle$ and $\left|\Phi_{1}\right\rangle$, two polarization-independent 50:50 beam splitters were used to split the four photons into the four modes $a, b, c$, and $d$. Next, the photons were sent through the quantum channel, where the noisy environment was simulated by a combination of birefringent quarter- (QWP) and half- (HWP) wave plates in each arm. The polarization analysis was performed using fur-

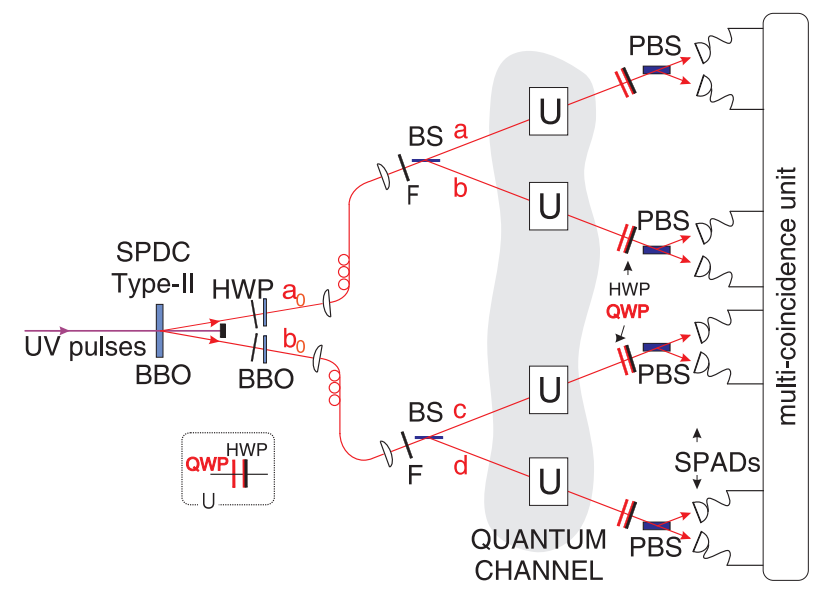

FIG. 1: Experimental setup to show the invariance of fourphoton entangled states under collective noise. The photons are emitted from spontaneous parametric down-conversion in a BBO crystal followed by birefringence compensation into two spatial modes $a_{0}$ and $b_{0}$. They are distributed into the four modes $a, b, c, d$ by 50:50 beam splitters (BS) behind interference filters $(\mathrm{F})$. The noisy quantum channel causing the unitary transformation $U^{\otimes 4}=U \otimes U \otimes U \otimes U$ is simulated by equal combinations of quarter- (QWP) and half-wave plates (HWP). Additional waveplates and polarizing beamsplitters (PBS) are employed for the polarization analysis of the four photons. For the registration of the decoherence-free states, events are selected where one photon was detected by singlephoton avalanche diodes (SPAD) in each of the four modes. 


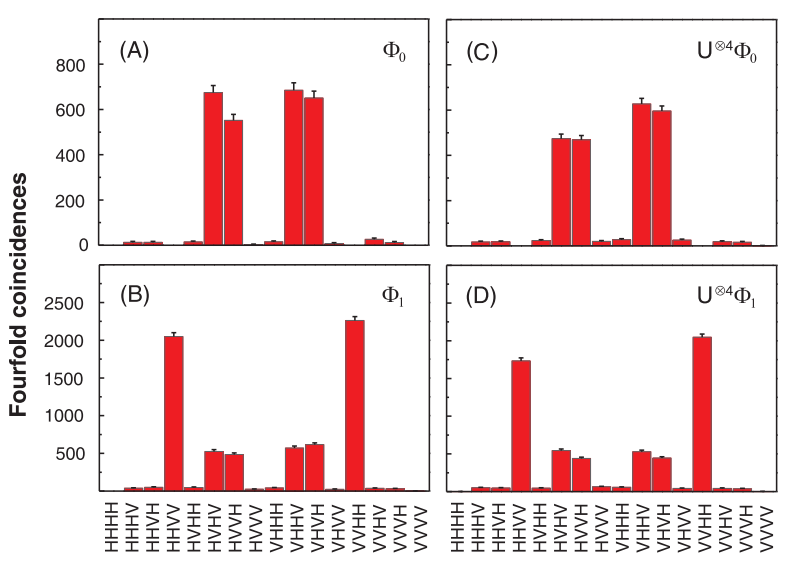

FIG. 2: Total counts of all the 16 possible fourfold detection events in $4 \mathrm{~h}$ measurement time for the four-photon states $\left|\Phi_{0}\right\rangle(\mathrm{A})$ and $\left|\Phi_{1}\right\rangle(\mathrm{B})$. Labels $\mathrm{H}$ and $\mathrm{V}$ indicate the horizontal and vertical polarization measurement outcomes for the four photons. (C) and (D) show the results in the presence of collective noise, i.e., under the same unitary transformation $U$. Here and in the following, $U$ was arbitrarily chosen and set with a HWP at an angle of $59^{\circ}$ and a QWP at an angle of $13.5^{\circ}$.

ther waveplates and polarizing beam splitters followed by silicon avalanche single photon detectors. Only events with one photon detected in each of the four arms have been selected.

Figure 2 shows the 16 possible fourfold coincidences for polarization analysis of one photon in each of the four outputs of the beam splitters exhibiting the characteristic statistics of the states $\left|\Phi_{0}\right\rangle(\mathrm{A})$ and $\left|\Phi_{1}\right\rangle(\mathrm{B})$. As a measure of the quality of the state preparation we use the quantum bit error rate (QBER), which is defined as the ratio of false events over total events or, in terms of the four-photon visibility $V$ [1] , as QBER $=(1-V) / 2$. For the data shown, we obtain QBER $=3.91 \% \pm 0.44 \%$ (A) and $\mathrm{QBER}=4.30 \% \pm 0.25 \%(\mathrm{~B})$. The ratio of total events observed upon encoding $\left|\Phi_{1}\right\rangle$ and $\left|\Phi_{0}\right\rangle$ is expected to be 3 for otherwise similar pumping conditions. Within the variation in the observed four-photon rate, this ratio is also reflected in the experiment.

To demonstrate the invariance of the four-photon states $\left|\Phi_{0}\right\rangle$ and $\left|\Phi_{1}\right\rangle$ under collective decoherence, i.e., under phase and bit flip errors caused by a birefringent quantum channel, we have arbitrarily chosen the unitary transformation $U=0.012 i \mathbb{1}-0.332 \sigma_{z}-0.707 \sigma_{y}+0.624 \sigma_{x}$. We implement this unitary transformation here by the addition of a HWP set at an angle of $59^{\circ}$ and a QWP set at an angle of $13.5^{\circ}$.

Figures 2(C) and 2(D) show that the distribution of detection events is not changed for the states $\left|\Phi_{0}\right\rangle$ and $\left|\Phi_{1}\right\rangle$ under the unitary transformation $U^{\otimes 4}$ (i.e., when the four photons are subject to collective noise). Here, we obtain similar error rates, with QBER $=7.11 \% \pm 0.50 \%$ $(\mathrm{C})$, and $\mathrm{QBER}=6.41 \% \pm 0.28 \%(\mathrm{D})$ for $\left|\Phi_{0}\right\rangle$ and $\left|\Phi_{1}\right\rangle$,

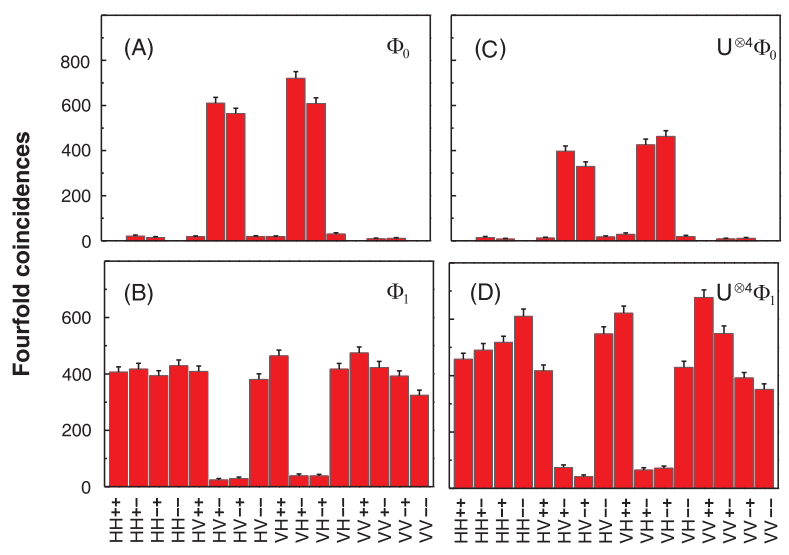

FIG. 3: Fourfold coincidences distinguishing between the states $\left|\Phi_{0}\right\rangle$ and $\left|\Phi_{1}\right\rangle$ by local polarization measurements on the four photons (counts in $4 \mathrm{~h}$ ). The photon polarization is analyzed in the $\{H, V\}$ and $\{+,-\}$ (i.e., $\pm 45^{\circ}$ basis) for the photons in paths $(a, b)$ and $(c, d)$, respectively. As before, (A) and (B) show the results of the analysis of the states $\left|\Phi_{0}\right\rangle$ and $\left|\Phi_{1}\right\rangle$ without noise, whereas (C) and (D) are the results in the presence of the collective noise $U^{\otimes 4}$.

respectively. From these measurements one can deduce the diagonal elements of the four-photon density matrices. Obviously, no additional elements are populated under the action of the collective noise indicating that the states $\left|\Phi_{0}\right\rangle$ and $\left|\Phi_{1}\right\rangle$ do not leave the DF subspace.

To distinguish between the states $\left|\Phi_{0}\right\rangle$ and $\left|\Phi_{1}\right\rangle$ by local measurements, we have projected the photon polarizations in paths $a$ and $b$ on the $\{H, V\}$ basis, and in paths $c$ and $d$ on the $\{+,-\}$, i.e., $\pm 45^{\circ}$ polarization basis. Figures 3(A) and 3(B) show the fourfold coincidence counts corresponding to a detection of $\left|\Phi_{0}\right\rangle$ and $\left|\Phi_{1}\right\rangle$, respectively, for a noiseless environment. For $\left|\Phi_{1}\right\rangle$, we clearly observe that the false fourfold coincidence counts, i.e., the terms $H V+-, H V-+, V H+-$, and $V H-+$, are negligible compared to the other terms, and vice versa for $\left|\Phi_{0}\right\rangle$. We observe an error rate of QBER $=5.23 \% \pm 0.46 \%$ for $\left|\Phi_{0}\right\rangle(\mathrm{A})$ and $\mathrm{QBER}=2.56 \% \pm 0.22 \%$ for $\left|\Phi_{1}\right\rangle(\mathrm{B})$. Analyzing the DF states in presence of the collective noise $U^{\otimes 4}$ shows that one is still able to distinguish reliably the two DF states, now with QBER $=6.82 \% \pm 0.75 \%(\mathrm{C})$ and $\mathrm{QBER}=3.99 \% \pm 0.26 \%(\mathrm{D})$, respectively.

In order to encode any arbitrary logical qubit, one could use two sources, one for $\left|\Phi_{0}\right\rangle$ and the other for $\left|\Phi_{1}\right\rangle$, and coherently overlap the generated photons. Yet, the technical requirements go beyond a first proof of principle. For a demonstration of the invariance of a logical qubit encoded in DF states, we prepared the state $\left|\Psi_{L}\right\rangle=\left(\sqrt{3}\left|\Phi_{0}\right\rangle-\left|\Phi_{1}\right\rangle\right) / 2$ and performed quantum state tomography of the encoded qubit before and after passage through a noisy quantum channel. We choose this state as it can be also obtained from the set-up shown in Fig. 1 by swapping modes $b \leftrightarrow c$. Figure 4 shows the elements of the density matrices $\rho_{\text {in }}$ (A) and $\rho_{\text {out }}(\mathrm{B})$ in 

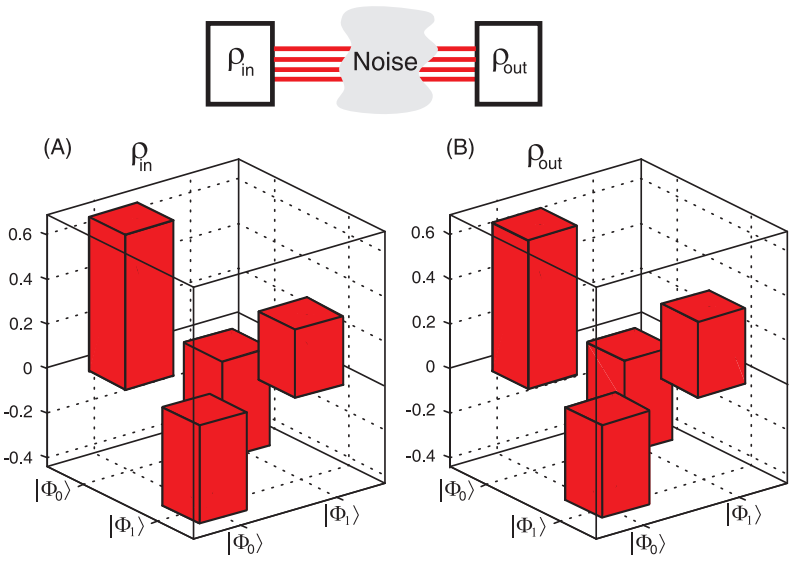

FIG. 4: Propagation of the logical qubit $\left|\Psi_{L}\right\rangle=\left(\sqrt{3}\left|\Phi_{0}\right\rangle-\right.$ $\left.\left|\Phi_{1}\right\rangle\right) / 2$ : (A) and (B) show the experimentally obtained density matrices before $\left(\rho_{\text {in }}\right)$ and after $\left(\rho_{\text {out }}\right)$ passage through a noisy quantum channel. The encoding in a DF subspace protected the transmission, leading to a fidelity of $F_{\rho_{\text {in }}, \rho_{\text {out }}}=$ $0.9958 \pm 0.0759$ in the presence of noise (overall measurement time $12 \mathrm{~h})$.

the $\left\{\left|\Phi_{0}\right\rangle,\left|\Phi_{1}\right\rangle\right\}$ basis of the logical qubit. The imaginary parts of the density matrices obtained are negligible. These results show that the diagonal elements before and after the interaction are in good agreement, and that the relative phase between basis states $\left|\Phi_{0}\right\rangle$ and $\left|\Phi_{1}\right\rangle$ is conserved. The quality of preparation of $\rho_{\text {in }}$ into the desired state $\rho_{L}=\left|\Phi_{L}\right\rangle\left\langle\Phi_{L}\right|$ is characterized by the fidelity $F_{\rho_{\text {in }}, \rho_{L}}=\operatorname{Tr}\left[\left(\sqrt{\rho_{L}} \rho_{\text {in }} \sqrt{\rho_{L}}\right)^{1 / 2}\right]$, where we obtain an experimental value of $F_{\rho_{\text {in }}, \rho_{L}}=0.989 \pm 0.038$. After exposing the state to the collective noise $U^{\otimes 4}$ we obtain an overlap between the initial and the outgoing state $F_{\rho_{\text {in }}, \rho_{\text {out }}}=0.996 \pm 0.076$, showing that the quantum information encoded in a DF subspace is preserved.

In addition, we want to point out that besides protecting against collective noise, the DF states are useful also for the communication of quantum information between two observers who do not share a common reference frame 13. In such a scenario, any realignment of the receiver's reference frame corresponds to the application of the same unitary transformation to each of the qubits which were sent. Yet, such an operation does not affect $\left|\Phi_{0}\right\rangle,\left|\Phi_{1}\right\rangle$, or any superposition thereof. Therefore, it is irrelevant whether or not the receiver's reference frame is aligned with the sender's reference frame in order to read the quantum information encoded in the DF states. Let us assume that the misalignment between sender and receiver is just given by the unitary transformation $U$. In this case the results shown in Fig. 3 and Fig. 4 clearly demonstrate that the receiver obtains the correct quantum information even if his reference frame does not coincide with the one of the sender.

To summarize, we experimentally demonstrated that SPDC can directly produce four-photon entangled states required to encode quantum information in decoherencefree subspaces and to protect it against collective noise. The quantum information encoded in the DF subspace is accessible by local measurements of the four photons, without two-qubit quantum logic gates being necessary, and thus realizable with state-of-the-art technology. This is relevant for possible applications of quantum communication [14]. We have performed a tomographic reconstruction of the density matrix of a logical qubit encoded in the DF subspace showing its immunity against a noisy environment. Our measurements also show that DF states permit the communication of quantum information even if the sender and the receiver do not share a reference frame. This is of great importance for future experiments studying quantum nonlocal effects between distant observers [13, 15].

This work was supported by the DFG (We 2451/1-2), the EU-Project RamboQ (IST-2001-38864), the Spanish MCYT Project BFM2002-02815, and the ESF.

[1] C.H. Bennett and D.P. DiVincenzo, Nature (London) 404, 247 (2000); M.A. Nielsen and I.L. Chuang, Quantum Computation and Quantum Information (Cambridge University Press, Cambridge, 2000).

[2] W.H. Zurek, Phys. Today 44, No. 10, 36 (1991).

[3] P.W. Shor, Phys. Rev. A 52, 2493 (1995); A.M. Steane, Phys. Rev. Lett. 77, 793 (1996); C.H. Bennett et al., Phys. Rev. A 54, 3824 (1996); R. Laflamme et al., Phys. Rev. Lett. 77, 198 (1996).

[4] G.M. Palma, K. Suominen, and A.K. Ekert, Proc. R. Soc. London, Ser. A 452, 567 (1996); L.-M. Duan and G.-C. Guo, Phys. Rev. Lett. 79, 1953 (1997).

[5] P. Zanardi and M. Rasetti, Phys. Rev. Lett. 79, 3306 (1997).

[6] D.A. Lidar, I.L. Chuang, and K.B. Whaley, Phys. Rev. Lett. 81, 2594 (1998); D.A. Lidar et al., Phys. Rev. A 61, 052307 (2000).

[7] J. Kempe et al., Phys. Rev. A 63, 042307 (2001).

[8] P.G. Kwiat et al., Science 290, 498 (2000); D. Kielpinski et al., Science 291, 1013 (2001); E.M. Fortunato et al., New. J. Phys 4, 5 (2002); J.E. Ollerenshaw, D.A. Lidar, and L.E. Kay, Phys. Rev. Lett. 91, 217904 (2003).

[9] E. Knill, R. Laflamme, and L. Viola, Phys. Rev. Lett. 84, 2525 (2000); L. Viola et al., Science 293, 2059 (2001).

[10] H. Weinfurter and M. Żukowski, Phys. Rev. A 64, 010102 (2001).

[11] M. Eibl et al., C. Kurtsiefer, M. Żukowski, \& H. Weinfurter, Phys. Rev. Lett. 90, 200403 (2003).

[12] P.G. Kwiat et al., Phys. Rev. Lett. 75, 4337 (1995).

[13] S.D. Bartlett, T. Rudolph, and R.W. Spekkens, Phys. Rev. Lett. 91, 027901 (2003).

[14] J.-C. Boileau, D. Gottesman, R. Laflamme, D. Poulin, and R.W. Spekkens, Phys. Rev. Lett. 92, 017901 (2004).

[15] A. Cabello, Phys. Rev. A 68, 042104 (2003); Phys. Rev. Lett. 91, 230403 (2003). 